end, the preservation of our culture, our intellectual heritage. And if there is a single, overarching responsibility of librarians in the next decades, it is to protect, and extend that fundamental mission in a time of growing complexity and confrontation.

The essential obligations of the Academy have not changed, but we must renew our society's understanding of them. The job will tax our imaginations as never before. But the answers will not be found within the rush of technological change nor the explosion of information that seems to dominate our lives. They will come from the processes of reason and dialogue that must always characterize our institutions. The need for libraries to reaffirm their central cultural and academic role will never be greater. And we, who have the ultimate responsibility for the health of our libraries, must help and support them.

\title{
Computer literacy and the mentally ill
}

\author{
By Josephine King Evans \\ Director, Florida Mental Health Institute Library \\ University of South Florida
}

\section{The computer as a therapeutic device.}

$\mathbf{T}$ eaching microcomputer skills to college and university students has become a new role for academic librarians, but during 1987, research center library staff at the University of South Florida in Tampa provided computer literacy to a different audience: the mentally ill. ${ }^{1}$ Located on the university campus, the Florida Mental Health Institute (FMHI) is the first state-assisted agency to implement such a program. Although there have been other automation projects in the mental health field, none has involved computer literacy for patients in a library setting. ${ }^{2}$

${ }^{1}$ Linda J. Piele, Judith Pryor, and Harold W. Tuckett, "Teaching Microcomputer Literacy: New Roles for Academic Librarians," College \& Research Libraries 47 (July 1986): 374-78.

${ }^{2}$ James L. Hedlund, Bruce W. Vieweg, and Dong W. Cho, "Mental Health Computing in the 1980s: I. General Information Systems and Clinical Documentation," Computers in Human Services 1 (Spring 1985): 3-33; James L. Hedlund, Bruce W. Vieweg, and Dong W. Cho, "Mental Health Computing in the 1980s: II. Clinical Applications," Computers in Human Services 1 (Sum-
Directed by Jack Zusman, FMHI is a University of South Florida research center that develops new treatment strategies and provides modernized training to strengthen mental health services throughout the state. Small, on-site model demonstration units employing behavior modification, family therapy, rehabilitation and other modes of treatment serve clients ranging in age from prekindergarten to the elderly. The average patient stay is ninety days. It was this population of approximately 600 people that the computer literacy program served during 1987.

Planned and implemented by the staff of the FMHI Research Library, the project was based in the smaller patient library located nearby; it was

mer 1985): 1-31; James E. Clark, Ann K. Lanphear, and Carol C. Riddick, "The Effects of Videogame Playing on the Response Selection Process of Elderly Adults," Journal of Gerontology 42 (1987): 82-85; Ellen Bouchard Ryan, "Memory for Goblins: A Computer Game for Assessing and Training Working Memory Skill," Clinical Gerontologist 6 (1986): 64-67. 
the first objective of a long-range goal to totally automate this collection used by patient-patrons. Subsequent objectives scheduled for future dates include both automated circulation and an online catalog.

Partially funded with a Library Services and Construction Act Title I Grant of $\$ 4,000$ from the State Library of Florida, the project was supplemented with the wages of two part-time library employees and a portion of the librarian's salary which made the total cost $\$ 12,100$. Expenditures were: IBM XT personal computer, color monitor, and IBM ProPrinter, \$3,032; supplies and software, $\$ 968$; wages and salary, $\$ 8,100$; TOTAL, $\$ 12,100$.

Selection of software remained experimental and continuous throughout the project as the library staff learned from observation what clients preferred. Micro Software Evaluations, a publication that includes prices, addresses of publishers, and hardware requirements, was useful. ${ }^{3}$ Public domain software may be nominally priced when a formatted diskette accompanies the request. Several publications list these computer programs. ${ }^{4}$ Other sources of information are local PC users groups. ${ }^{5}$

Necessary training for the project included selfstudy exercises completed by the librarian, who subsequently taught library staff basic computer skills such as use of DOS, concept of memory function, operation of both keyboard and printer, and how to access various software. Once knowledgeable about the hardware, staff members examined programs to become familiar with their complexity and modes of access. To simplify use, library staff affixed basic directions for programs to the sleeves of diskettes. Because training was interspersed with regular library duties, staff time of 360 hours for the three-month learning period is an estimate only.

Before implementation of the project, the librarian established guidelines; the computer was to be utilized by clients and only with the intended software. Library administrative use was not permitted. Although occasionally clients come to the library individually, visiting in groups is the norm. After briefly orienting clients to basic computer use, library staff demonstrated software. Because many people (mentally ill or not) fear the unknown, it was important to relate the initial com-

\footnotetext{
${ }^{3}$ The Florida Center for Instructional Computing: Micro Software Evaluations (Tampa: College of Education, University of South Florida, 1986).

${ }^{4}$ Bertram Gader, Free Software for the IBM PC (New York: Warner Software, 1984); PC-SIG Library: Public Domain and User-Supported Software for the IBM PC, 4th ed. (Sunnyvale, Calif.: PC-Sig, 1987); Robert Froelich, The IBM PC (and Compatible) Free Software Catalog and Directory, lst ed. (New York: Dilithium Press, 1986).

${ }^{5}$ The Pinellas PC Users Group, Inc., 3401 Gandy Boulevard, \#628, Pinellas Park, FL 33565.
}

puter exposure to something familiar to participants. Appropriate introductory programs were Pinball and Pac Man, variations of arcade games. Another consideration was the success factor. If a participant had failed in the first attempt, that individual might have been too discouraged to try again.

After the initial fear of the computer subsided, clients selected more complex software. Adolescents and young adults favored decision-making situations such as those in Executive Suite; the participant is first a job applicant, then an employee faced with career decisions. Elderly adults, particularly those in memory therapy, requested programs that stimulate recall, such as States 'N Caps; the participant supplies the capitals of states for an on-screen map of the U.S.A. Stimulated by these experiences, patients inquired about word processing and the availability of software that would produce newsletters and flyers; these requests indicate that there may be a sizable receptive audience for such programs.

Evaluation of the FMHI Library Computer Literacy Project was accomplished objectively with both library attendance statistics and with an informal, brief questionnaire developed by the librarian with the guidance of clinical staff. Statistics document an increase in attendance of $52 \%$ over a similar period before the program began. Patient responses to the questionnaire were positive, and even those who had not used the computer indicated a desire to do so at a future time. Other subjective evaluation was provided by the library staff who rated the usefulness of the software.

As significant as what software clients favored was the unique manner in which they used the computer. Usually considered a piece of equipment for the individual, the FMHI Client Library computer was seldom utilized in that manner; patients preferred, instead, group involvement. Participants consulted each other about responses and took turns operating the keyboard. As they cooperated, the various distinct individuals developed into supportive team-members who offered praise to each other for correct responses and sympathy for incorrect ones.

Library staff had anticipated problems with sharing by users, but such difficulties never arose. Instead, there was obvious enjoyment of mentally ill persons in participating as a group with the computer. This unique use by clients at the Florida Mental Health Institute Client Library indicates that in the hands of qualified mental health practitioners, computers may have positive social applications in therapy.

Based upon the favorable reception by mentally ill clients of this computer literacy project at the University of South Florida-Florida Mental Health Institute, the librarian has requested additional LSCA Grant funding to proceed with automated circulation and an online catalog for the FMHI Patient Library in 1989. 


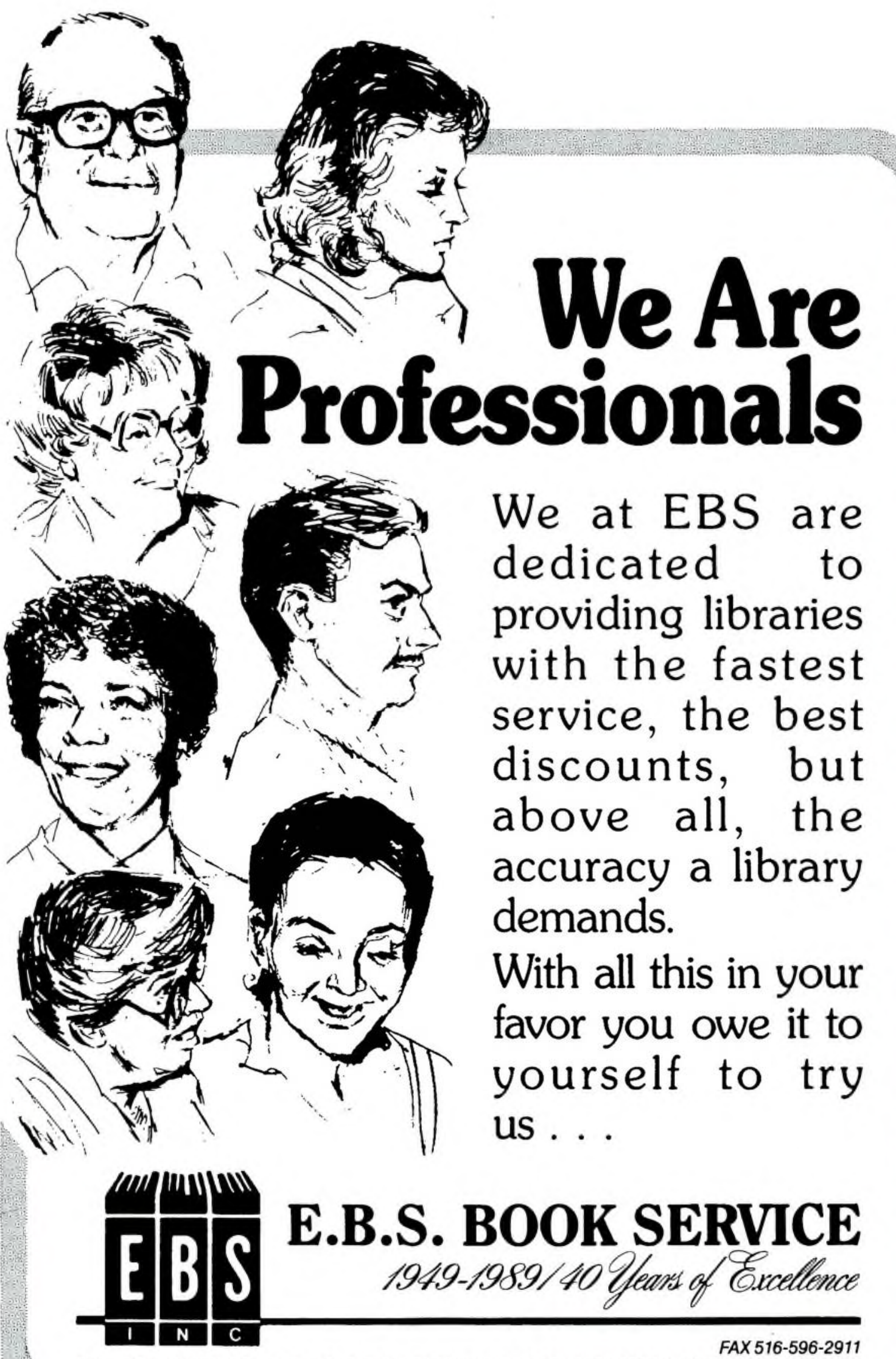

E.B.S. INC. BOOK SERVICE - 290 BROADWAY, LYNBROOK, NEW YORK 11563 - 516-593-1207 